\title{
Sustainable High Yield Route to Cellulose Nanocrystals from Bacterial Cellulose
}

\section{Pääkkönen, Timo}

2019-09-03

Pääkkönen , T , Spiliopoulos , P , Nonappa , D , Kontturi , K S , Penttilä , P , Viljanen , M , Svedström , K \& Kontturi , E 2019, ' Sustainable High Yield Route to Cellulose Nanocrystals from Bacterial Cellulose ' , ACS Sustainable Chemistry \& Engineering , vol. 7 , no. 17 , pp. 14384-14388 . https://doi.org/10.1021/acssuschemeng.9b04005

http://hdl.handle.net/10138/306076

https://doi.org/10.1021/acssuschemeng.9b04005

cc_by

publishedVersion

Downloaded from Helda, University of Helsinki institutional repository.

This is an electronic reprint of the original article.

This reprint may differ from the original in pagination and typographic detail.

Please cite the original version. 


\section{Sustainable High Yield Route to Cellulose Nanocrystals from Bacterial Cellulose}

Timo Päakkönen, ${ }^{\dagger}$ Panagiotis Spiliopoulos, ${ }^{\dagger}$ Nonappa, ${ }^{\ddagger}$ Katri S. Kontturi, ${ }^{\S}, \|$ Paavo Penttilä, ${ }^{\dagger}$ Mira Viljanen, ${ }^{\perp}$ Kirsi Svedström, ${ }^{\perp}$ and Eero Kontturi* ${ }^{*}{ }^{\dagger}$

${ }^{\dagger}$ Department of Bioproducts and Biosystems, School of Chemical Engineering, Aalto University, P.O. Box 16300, 00076 Aalto, Finland

${ }^{\ddagger}$ Department of Applied Physics, School of Science, Aalto University, P.O. Box 15100, 00076 Aalto, Finland

${ }^{\S}$ VTT Technical Research Centre of Finland, P.O. Box 1000, FI-02044 VTT, Finland

"Polymer and Composites Engineering (PaCE) group, Institute of Materials Chemistry and Research, University of Vienna,

Währingerstrasse 42, 1090 Vienna, Austria

${ }^{\perp}$ Department of Physics, University of Helsinki, P.O. Box 64, FI-00014 Helsinki, Finland

\section{Supporting Information}

ABSTRACT: $\mathrm{HCl}$ gas hydrolysis of a bacterial cellulose (BC) aerogel followed by 2,2,6,6-tetramethylpiperidine-1-oxyl radical-mediated oxidation was used to produce hydrolyzed BC with carboxylate groups, which subsequently disintegrated into a stable dispersion of cellulose nanocrystals (CNCs). The degree of polymerization was successfully reduced from 2160 to 220 with a CNC yield of $>80 \%$.

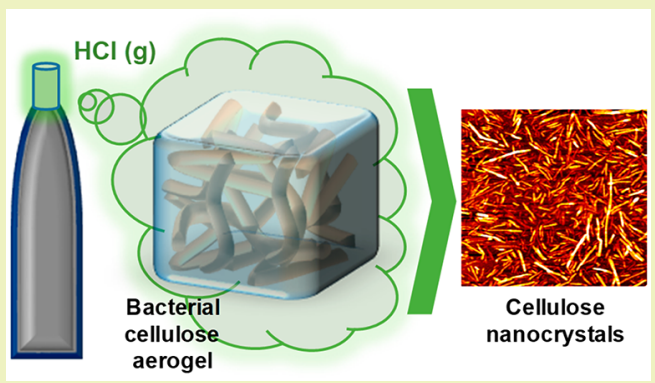

KEYWORDS: Colloidal dispersion, Gaseous acid, Hydrolysis, Nanocellulose, TEMPO-oxidation

\section{INTRODUCTION}

Cellulose nanocrystals (CNCs), isolated from plant-based fibers or a microbial cultivation product, are a premium example of a modern renewable nanomaterial. ${ }^{1}$ Aside from its fully biobased origin, it is biodegradable, nontoxic, and possesses chirality as well as high mechanical strength. ${ }^{2}$ Proposed applications for CNCs include composites, ${ }^{3}$ chiral templates, ${ }^{4}$ biomedical devices, ${ }^{5}$ and responsive materials, ${ }^{6}$ to name a few. However, the sustainable aspect of CNCs is compromised by its production, generally performed with ca. $65 \%$ aqueous sulfuric acid, leading to difficult purification procedures, high water consumption, and poor yields. ${ }^{7-10}$ To tackle these problems, our group recently introduced a method that utilizes $\mathrm{HCl}$ vapor $^{11}$ or gas ${ }^{12}$ for cellulose hydrolysis and subsequent $\mathrm{CNC}$ manufacturing. The approach has several advantages: it leaves solid fibers as a product and they are far easier to purify than an aqueous reaction mixture, resulting in higher yields and minimal water consumption. ${ }^{12}$ Moreover, the gaseous acid is easier to recover and recycle than a liquid/solid reaction product. On the other hand, the major problem with the $\mathrm{HCl}$ gas process lies in the difficult dispersion of uncharged $\mathrm{CNCs}$ from the already hydrolyzed fiber matrix. A proof-ofconcept with dispersion in formic acid exhibited sizable CNC yields of $>97 \%$ but formic acid with heavy (tens of hours) sonication is scarcely a viable dispersion medium even under laboratory conditions. ${ }^{11}$ Attempts with alternative dispersion methods have failed to reach the remarkable yields. ${ }^{13,14}$ Here, we want to communicate a straightforward way to utilize $\mathrm{HCl}$ gas for manufacturing CNCs in high yields from a commercially available bacterial cellulose (BC) substrate. Although more than often quoted to be highly crystalline, $\mathrm{BC}$ has been shown to possess a so-called leveling-off degree of polymerization (LODP) ${ }^{15}$ and it also has been utilized in CNC preparation. ${ }^{16,17}$ The actual isolation of CNCs was facilitated by a second step with oxidation catalyzed by $\mathrm{N}$-oxoammonium cation of 2,2,6,6- tetramethylpiperidine-1-oxyl (TEMPO) that imparts charge on the CNC surface, enabling aqueous dispersion of CNCs.

\section{RESULTS AND DISCUSSION}

The full procedure for obtaining CNCs from BC is depicted in Figure 1. Unlike hierarchically arranged cellulose microfibrils in plant cell walls, BC is readily excreted by certain microbes into an isotropic network of microfibrils, forming a hydrogel of pure cellulose with high accessibility. First, the BC cubes intended for dessert foodstuff were washed with mild alkali to remove sugars and other taste ingredients to obtain a pure BC hydrogel

Received: July 12, 2019

Revised: August 14, 2019

Published: August 16, 2019 
[1]

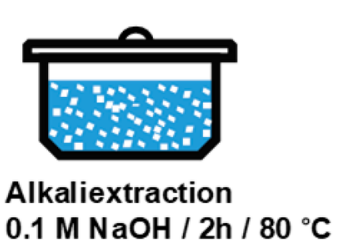

$[2]$

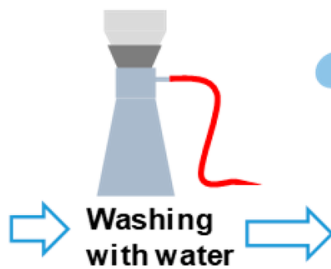

[3]

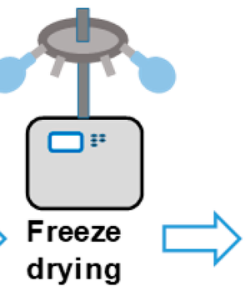

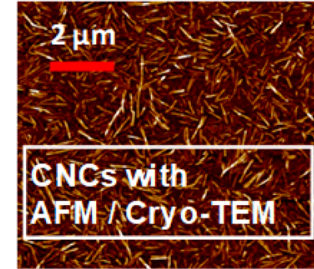

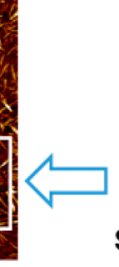

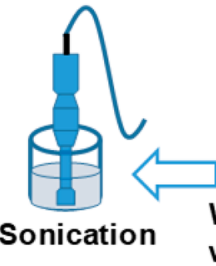

[7]

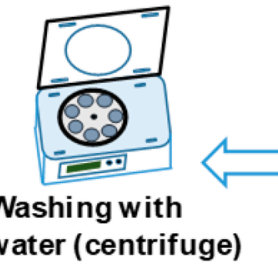

[6]
[4]

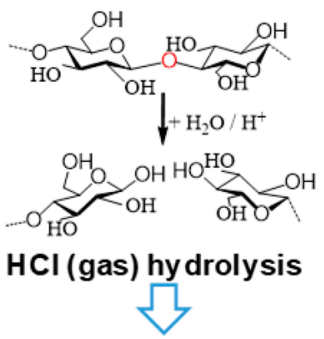

Washing with water

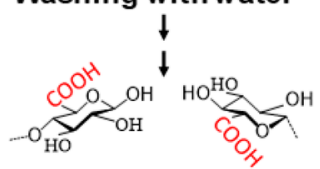

TEMPO-mediated oxidation $\mathrm{RT}$, aqueous conditions $6 \mathrm{mmol} \mathrm{NaOCl} / \mathrm{g} \mathrm{BC}$

[8]

Figure 1. Scheme of the CNC production process.

(see Figure $2 a-c$ for visual appearance). The following drying step from hydrogel into aerogel was crucial: freeze-drying had

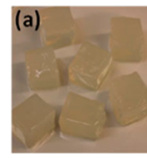

Untreated

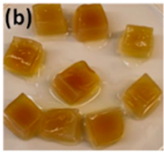

During cooking Purified

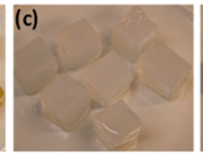

Freeze dried Freeze dried (Succesfull) (Failed)

Figure 2. Pretreatment of bacterial cellulose. Bacterial cellulose (a) was washed with pure water, extracted (b) with alkali $(0.1 \mathrm{M} \mathrm{NaOH}$, $85{ }^{\circ} \mathrm{C}, 3 \mathrm{~h}$ ), washed again with pure water (c), and freeze-dried (d) without prior freezing. Freeze-dried BC with prior freezing (e) collapsed with loss in accessibility.

to be performed to pristine, wet $\mathrm{BC}$, which was then frozen by the emerging vacuum during the first seconds of the process (Figure 2d, Figure S2). Usually in freeze-drying, the sample is frozen before placing it in the vacuum. However, when a wet, unfrozen sample is positioned under a vacuum of a freeze-drier, it freezes almost immediately due to three different factors: (i) depression of the melting point, (ii) evaporative cooling of the sample as the water immediately starts to vaporize, and (iii) a compressive decrease of temperature as pressure is decreased. This resulted in Brunauer-Emmett-Teller (BET) surface area of $29 \mathrm{~m}^{2} / \mathrm{g}$ (Figure S5). By contrast, when the sample was frozen before placing it in a freeze-dryer, as is the case in conventional freeze-drying, the surface area was far smaller (BET area $16 \mathrm{~m}^{2} / \mathrm{g}$, Figures S2, S6). This can also be visually observed from Figure 2e. Furthermore, merging several cubes together by blending and subsequent drying led to severe loss in accessibility, as attested by a weak hydrolysis effect (see Figure 3). The reason behind the distinctions in the freezing methods (Figure $2 \mathrm{~d}$ vs $2 \mathrm{e}$ ) may lie in different routes to crystallization of water but a systematic study is outside the scope of this letter.

Purified and freeze-dried bacterial cellulose (BC) cubes were hydrolyzed (dry matter content 94\%) with $\mathrm{HCl}$ gas (1 bar pressure). The degree of polymerization (DP) was decreased

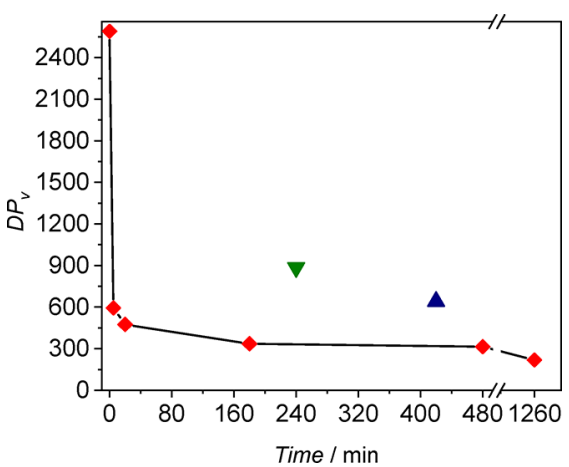

Figure 3. DP development upon exposure to 1 bar $\mathrm{HCl}(\mathrm{g})$. Symbols denote freeze-dried BC with $(\boldsymbol{A})$ and without $(\checkmark)$ prior freezing, BC mixed in kitchen blender and dried in fumehood $(\boldsymbol{\nabla})$.

from 2590 to 220 over $21 \mathrm{~h}$ (Figure 3), indicating the cleavage of disordered cellulose between the crystallites in semicrystalline cellulose microfibrils, i.e., LODP, necessary for CNC isolation. This DP decrease correlates well with the LODP value reported earlier for BC. ${ }^{15}$ The yield of $21 \mathrm{~h}$ hydrolysis after rinsing with water was $86.8 \%$. Akin to the plant-based cellulose in fiber form, ${ }^{11}$ the bulk of the hydrolysis occurred within minutes of exposing the correctly dried $\mathrm{BC}$ to 1 bar $\mathrm{HCl}$ (g). Moreover, the BET surface area of BC increased from 29 to $52 \mathrm{~m}^{2} / \mathrm{g}$ upon hydrolysis (Figures S5, S7). After TEMPOmediated oxidation, the final product with a carboxylate content of $1.0 \mathrm{mmol} \mathrm{COOH} / \mathrm{g} \mathrm{BC}$ (Figure S1) turned to gel after purification. The zeta potential of the CNCs amounted to ca. $40 \mathrm{mV}$ (Table S2). The yield of TEMPO-mediated oxidation was $93.1 \%$, resulting in a total yield (hydrolysis and oxidation) of $80.8 \%$ after purification by centrifugation.

Hydrolyzed and TEMPO-oxidized BC was finally disintegrated into $\mathrm{CNCs}$ by sonication, as visualized with atomic force microscopy (AFM) imaging and transmission electron microscopy (TEM) (Figure 4a,b). To prevent aggregation artifacts, cryo-TEM images (Figure S3) were used for image analysis (Figure S4) which revealed rather narrow distributions of width and length (Figure 4c,d). Although a small number of 

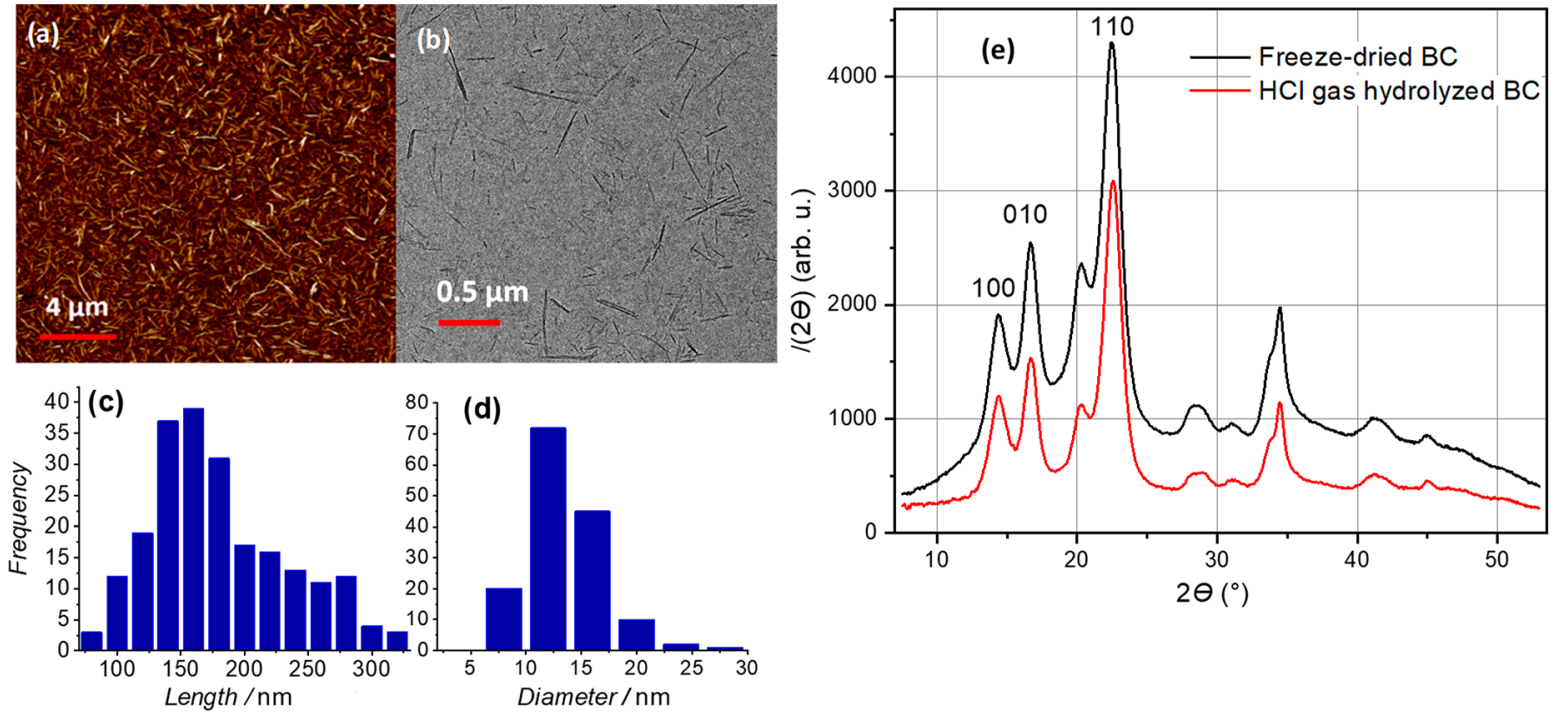

Figure 4. (a) AFM height image, (b) TEM image and dimensional (c) length and (d) diameter analysis from cryo-TEM images (Figures S3 and S4) of hydrolyzed, TEMPO-oxidized, and sonicated BC. (e) WAXS intensities of the freeze-dried and hydrolyzed (18 h, $1 \mathrm{bar}) \mathrm{BC}$ with the strongest diffraction peaks indexed according to cellulose $\mathrm{I}_{\alpha}$ (see Figure S8 for peak fitting).

longer fibrils could be discerned from the images, the vast majority of CNCs were within 100-300 nm length with the mean at $170 \mathrm{~nm}$, correlating fairly well with the DP value (Figure 3). The measured length for CNCs is somewhat shorter than in a number of previous accounts ${ }^{16,17}$ on BCbased CNCs, but these works have generally not presented statistical histograms on the CNC dimensions. Furthermore, the high yield and high charge due to TEMPO-oxidation may also affect the size distribution of the CNCs in this study.

It is curious that in $\mathrm{HCl}$ gas hydrolysis followed by TEMPOoxidation, the CNC yield here from BC (>80\%) is markedly higher than previously from plant-based (cotton) fibers $(\sim 50 \%) .{ }^{14}$ Because the DP reduction and carboxylic contents are roughly similar in both cases, the reason for the higher CNC yield must lie in the physical structure: the correctly chosen drying method. When the hydrolyzed BC was leached in water and the filtrate was analyzed with high performance anion exchange chromatography (HPAEC), the data showed that 13 wt \% glucose could be released from the substrate after hydrolysis (Table S3). This is 1 order of magnitude different from cotton-based fibers where few wt $\%$ of glucose could be leached from the fibers hydrolyzed under similar conditions. ${ }^{12}$ Overall, it appears that $\mathrm{HCl}$ gas hydrolysis on $\mathrm{BC}$ is faster and more efficient than on plant-based fibers.

A highly porous structure of the freeze-dried BC (Figure 2d) is also more accessible to cavitation during sonication. The hierarchical structure of the plant fiber is largely resistant to sonication, as shown earlier with TEMPO-oxidized microcrystalline cellulose (MCC) with maximal carboxylate contents where the yields stalled at a maximum of $20 \% .{ }^{18}$ By contrast, a more recent work with TEMPO-oxidation amounted to $70 \%$ yields from MCC, but only after intensive high-energy sonication for $>30 \mathrm{~min}$, which may prove to be a bottleneck in large scale implementation. ${ }^{19}$ In terms of other novel techniques, the $80 \%$ yield here from $\mathrm{BC}$ is also fair, compared to, e.g., electron beam irradiation (bleached softwood kraft pulp, yield 35-67\%), ${ }^{20}$ esterification (bleached eucalyptus kraft pulp, yield 25\%), ${ }^{21}$ TEMPO-mediated oxidation (microgranulars of cellulose from cotton, microcrystalline cellulose from cotton, yield $4-20 \%){ }^{22}$ acetylation in ionic liquids (wood, yield 44\%), ${ }^{23}$ ball milling with centrifugation (MCC, bleached kraft pulp, yield 20\%), ${ }^{24}$ hydrolysis with subcritical water (MCC, yield 22\%), ${ }^{25}$ ball milling and phosphotungstic acid hydrolysis (bamboo pulp, yield $88 \%),{ }^{26}$ and microwaveassisted hydrothermal treatment (orange peel waste, yield 30$68 \%) .{ }^{27}$ It must be emphasized that, in contrast to CNCs, cellulose nanofibril (CNF) yield from plant-based sources is in general very high, regularly around $80-90 \%$ for TEMPOoxidized fibers. ${ }^{28}$

Wide angle X-ray scattering (WAXS) data in Figure 4e and Table S1 indicate that the crystallinity index increased from $45 \%$ to $61 \%$, i.e., close to $30 \%$ upon hydrolysis of BC, which may play a role in the high yield of CNCs. This increase was far larger than the 10-15\% increment observed earlier with plant-based fibers. ${ }^{11}$ This increase is likely due to (i) crystallization of cellulose during $\mathrm{HCl}(\mathrm{g})$ hydrolysis, as elaborated previously, ${ }^{11}$ and (ii) removal of disordered cellulose during the washing step which removes the watersoluble sugars (glucose) after the hydrolysis. As for the allomorph ratio $\left(\mathrm{I}_{\alpha} / \mathrm{I}_{\beta}\right)$, it appeared to remain constant (see Supporting Information) despite the fact that a number of accounts report alterations in the ratio upon hydrolysis. ${ }^{29,30}$ We emphasize the texture of the BC remains unchanged after $\mathrm{HCl}(\mathrm{g})$ hydrolysis, thus omitting artifacts due to texture in WAXS analysis. For the possible thermodynamic mechanism behind the crystallization in $\mathrm{HCl}$ gas, see ref 11 .

In summary, $>80 \%$ CNC yield from BC with moderate sonication represents progress in sustainable $\mathrm{CNC}$ production. Here, utilizing a BC intended for foodstuffs is certainly not a viable route beyond demonstration but recent research has shown how $\mathrm{BC}$ can be produced from, e.g., agricultural waste products in large quantities. ${ }^{31}$ In terms of sustainability, the benefits can be outlined in three categories: (i) ca. 50\% higher yield of CNCs than with the usual liquid/solid procedure (ii) utilizing waste instead of wood pulp, the most common commercial source for CNCs, reduces the exploitation of forest resources as well as minimizes chemical and water consumption; (iii) dry gaseous acid can be easily recycled and it further minimizes the water consumption when purification procedures, such as dialysis in liquid/solid systems, can be 
replaced by controlled rinsing with moderate amounts of water as nearly all acid is removed by desorption after the hydrolysis. Indeed, the full recovery of aqueous acid has prevented sustainable, large scale use of acid in hydrolysis of cellulose in the past. ${ }^{32}$ Furthermore, the thermal stability of carboxylic groups should be higher than those of sulfate groups which are the norm for charged groups on $\mathrm{CNCs}{ }^{33}$

\section{ASSOCIATED CONTENT}

\section{S Supporting Information}

The Supporting Information is available free of charge on the ACS Publications website at DOI: 10.1021/acssuschemeng.9b04005.

Experimental details, conductometric titrations curves, SEM images, cryo TEM images and background for image analysis, BET raw data, WAXS data analysis, zeta potential analysis, HPAEC analysis (PDF)

\section{AUTHOR INFORMATION}

\section{Corresponding Author}

*E. Kontturi. E-mail: eero.kontturi@aalto.fi; Tel.: +358 50 3449278 .

ORCID $\odot$

Nonappa: 0000-0002-6804-4128

Paavo Penttilä: 0000-0003-0584-4918

Eero Kontturi: 0000-0003-1690-5288

\section{Funding}

P.P. acknowledges Academy of Finland (Grant no. 315768) and T.P. Acknowledges Academy of Finland (Grant no. 300364).

\section{Notes}

The authors declare no competing financial interest.

\section{ACKNOWLEDGMENTS}

We acknowledge Ms. Suvi Muukka and Ms. Xuetong Shi for auxiliary laboratory work with $\mathrm{HCl}$ gas hydrolysis and TEMPO-mediated oxidation. We acknowledge Ms. Tuyen Nguyen for auxiliary laboratory work with zeta potential measurement. We acknowledge Ms. Yingfeng Wang for auxiliary laboratory work with HPAEC analysis. T.P. acknowledges Academy of Finland (Grant no. 300364) for financial support. HYBER Centre of Excellence (2014-2019) and the Aalto University Nanomicroscopy Center (Aalto-NMC) are acknowledged for the use of its facilities. Moreover, E.K. is grateful for the support by the FinnCERES Materials Bioeconomy Ecosystem.

\section{REFERENCES}

(1) Habibi, Y.; Lucia, L. A.; Rojas, O. J. Cellulose nanocrystals: chemistry, self-assembly, and applications. Chem. Rev. 2010, 110, 3479-3500.

(2) Eichhorn, S. J. Stiff as a Board: Perspectives on the Crystalline Modulus of Cellulose. ACS Macro Lett. 2012, 1, 1237-1239.

(3) McKee, J. R.; Huokuna, J.; Martikainen, L.; Karesoja, M.; Nykänen, A.; Kontturi, E.; Tenhu, H.; Ruokolainen, J.; Ikkala, O. Molecular Engineering of Fracture Energy Dissipating Sacrificial Bonds Into Cellulose Nanocrystal Nanocomposites. Angew. Chem., Int. Ed. 2014, 53, 5049-5053.

(4) Kelly, J. A.; Giese, M.; Shopsowitz, K. E.; Hamad, W. Y.; MacLachlan, M. J. The Development of Chiral Nematic Mesoporous Materials. Acc. Chem. Res. 2014, 47, 1088-1096.
(5) Sheikhi, A.; Hayashi, J.; Eichenbaum, J.; Gutin, M.; Kuntjoro, N.; Khorsandi, D.; Khademhosseini, A. Recent advances in nanoengineering cellulose for cargo delivery. J. Controlled Release 2019, 294, 53-76.

(6) Kontturi, E.; Laaksonen, P.; Linder, M. B.; Nonappa; Gröschel, A. H.; Rojas, O. J.; Ikkala, O. Advanced Materials through Assembly of Nanocelluloses. Adv. Mater. 2018, 30, 1703779.

(7) Mukherjee, S. M.; Woods, H. J. X-ray and electron microscope studies of the degradation of cellulose by sulphuric acid. Biochim. Biophys. Acta 1953, 10, 499-511.

(8) Dong, X. M.; Revol, J.; Gray, D. G. Effect of microcrystallite preparation conditions on the formation of colloid crystals of cellulose. Cellulose 1998, 5, 19-32.

(9) Mariano, M.; El Kissi, N.; Dufresne, A. Cellulose nanocrystals and related nanocomposites: Review of some properties and challenges. J. Polym. Sci., Part B: Polym. Phys. 2014, 52, 791-806.

(10) Trache, D.; Hussin, M. H.; Haafiz, M. K. M.; Thakur, V. K. Recent progress in cellulose nanocrystals: sources and production. Nanoscale 2017, 9, 1763-1786.

(11) Kontturi, E.; Meriluoto, A.; Penttilä, P. A.; Baccile, N.; Malho, J.; Potthast, A.; Rosenau, T.; Ruokolainen, J.; Serimaa, R.; Laine, J.; Sixta, H. Degradation and Crystallization of Cellulose in Hydrogen Chloride Vapor for High-Yield Isolation of Cellulose Nanocrystals. Angew. Chem., Int. Ed. 2016, 55, 14455-14458.

(12) Pääkkönen, T.; Spiliopoulos, P.; Knuts, A.; Nieminen, K.; Johansson, L. S.; Enqvist, E.; Kontturi, E. From vapour to gas: Optimising cellulose degradation with gaseous $\mathrm{HCl}$. React. Chem. Eng. 2018, 3, 312-318.

(13) Fang, W.; Arola, S.; Malho, J.; Kontturi, E.; Linder, M. B.; Laaksonen, P. Noncovalent Dispersion and Functionalization of Cellulose Nanocrystals with Proteins and Polysaccharides. Biomacromolecules 2016, 17, 1458-1465.

(14) Lorenz, M.; Sattler, S.; Reza, M.; Bismarck, A.; Kontturi, E. Cellulose hydrolysis by acid vapour: towards a more effortless isolation of cellulose nanocrystals. Faraday Discuss. 2017, 202, 315330.

(15) Väljamäe, P.; Sild, V.; Nutt, A.; Pettersson, G.; Johansson, G. Acid hydrolysis of bacterial cellulose reveals different modes of synergistic action between cellobiohydrolase I and endoglucanase I. Eur. J. Biochem. 1999, 266, 327-334.

(16) Salari, M.; Khiabani, M. S.; Mokarrama, R. R.; Ghanbarzadeh, B.; Kafil, H. S. Preparation and characterization of cellulose nanocrystals from bacterial cellulose produced in sugar beet molasses and cheese whey media. Carbohydr. Polym. 2019, 122, 280-288.

(17) Winter, H. T.; Cerclier, C.; Delorme, N.; Bizot, H.; Quemener, B.; Cathala, B. Improved Colloidal Stability of Bacterial Cellulose Nanocrystal Suspensions for the Elaboration of Spin-Coated Cellulose-Based Model Surfaces. Biomacromolecules 2010, 11, 31443151.

(18) Salminen, R.; Reza, M.; Pääkkönen, T.; Peyre, J.; Kontturi, E. TEMPO-mediated oxidation of microcrystalline cellulose: limiting factors for cellulose nanocrystal yield. Cellulose 2017, 24, 1657-1667.

(19) Zhou, Y.; Saito, T.; Bergström, L.; Isogai, A. Acid-free preparation of cellulose nanocrystals by TEMPO oxidation and subsequent cavitation. Biomacromolecules 2018, 19, 633-639.

(20) Lee, M.; Heo, M. H.; Lee, H.; Lee, H.; Jeong, H.; Kim, Y.; Shin, J. Facile and eco-friendly extraction of cellulose nanocrystals via electron beam irradiation followed by high-pressure homogenization. Green Chem. 2018, 20, 2596-2610.

(21) Chen, L.; Zhu, J.; Baez, C.; Kitin, P.; Elder, T. Highly thermalstable and functional cellulose nanocrystals and nanofibrils produced using fully recyclable organic acids. Green Chem. 2016, 18, 38353843.

(22) Peyre, J.; Pääkkönen, T.; Reza, M.; Kontturi, E. Simultaneous preparation of cellulose nanocrystals and micron-sized porous colloidal particles of cellulose by TEMPO-mediated oxidation. Green Chem. 2015, 17, 808-811. 
(23) Abushammala, H.; Krossing, I.; Laborie, M. Ionic liquidmediated technology to produce cellulose nanocrystals directly from wood. Carbohydr. Polym. 2015, 134, 609-616.

(24) Kang, X.; Kuga, S.; Wang, C.; Zhao, Y.; Wu, M.; Huang, Y. Green Preparation of Cellulose Nanocrystal and Its Application. ACS Sustainable Chem. Eng. 2018, 6, 2954-2960.

(25) Novo, L. P.; Bras, J.; García, A.; Belgacem, N.; Curvelo, A. A. Subcritical water: a method for green production of cellulose nanocrystals. ACS Sustainable Chem. Eng. 2015, 3, 2839-2846.

(26) Lu, Q.; Cai, Z.; Lin, F.; Tang, L.; Wang, S.; Huang, B. Extraction of Cellulose Nanocrystals with a High Yield of $88 \%$ by Simultaneous Mechanochemical Activation and Phosphotungstic Acid Hydrolysis. ACS Sustainable Chem. Eng. 2016, 4, 2165-2172.

(27) Matharu, A. S.; de Melo, E. M.; Remón, J.; Wang, S.; Abdulina, A.; Kontturi, E. Processing of Citrus Nanostructured Cellulose: A Rigorous Design-of-Experiment Study of the Hydrothermal Microwave-Assisted Selective Scissoring Process. ChemSusChem 2018, 11, 1344-1353.

(28) Kuramae, R.; Saito, T.; Isogai, A. TEMPO-oxidized cellulose nanofibrils prepared from various plant holocelluloses. React. Funct. Polym. 2014, 85, 126-133.

(29) Hayashi, N.; Sugiyama, J.; Okano, T.; Ishihara, M. The enzymatic susceptibility of cellulose microfibrils of the algal-bacterial type and the cotton-ramie type. Carbohydr. Res. 1997, 305, 261-269.

(30) Wada, M.; Okano, T. Localization of $\mathrm{I}_{\alpha}$ and $\mathrm{I}_{\beta}$ phases in algal cellulose revealed by acid treatments. Cellulose 2001, 8, 183-188.

(31) Kongruang, S. Bacterial Cellulose Production by Acetobacter xylinum Strains from Agricultural Waste Products. Appl. Biochem. Biotechnol. 2008, 148, 245-256.

(32) Rinaldi, R.; Schüth, F. Acid Hydrolysis of Cellulose as the Entry Point into Biorefinery Schemes. ChemSusChem 2009, 2, 1096-1107.

(33) Camarero Espinosa, S.; Kuhnt, T.; Foster, E. J.; Weder, C. Isolation of Thermally Stable Cellulose Nanocrystals by Phosphoric Acid Hydrolysis. Biomacromolecules 2013, 14, 1223-1230. 